\title{
Recherche sur l'être humain: bon projet de loi, mais quelques bémols
}

La Commission nationale d'éthique pour la médecine humaine est favorable au projet de loi fédérale relative à la médecine humaine. Elle critique surtout la possibilité de contraindre des personnes incapables de discernement à se soumettre à des recherches. De plus, le travail des commissions cantonales ne devrait être pas limité à la surveillance et à l'examen des projets.

Commission nationale d'éthique pour la médecine humaine

Correspondance:

Dr iur. Sibylle Schürch Commission nationale d'éthique pour la médecine humaine OFSP

Seilerstrasse 8

$\mathrm{CH}-3003$ bern

Tél. 0313249365

Fax 0313226233

www.nek-cne.ch
La CNE salue le projet de légiférer dans le domaine de la recherche sur l'être humain et le fait que la Confédération devienne compétente en la matière. Selon elle, le projet de loi soumis est de bonne facture. La recherche débouche sur de nouvelles connaissances, qui sont essentielles pour la protection de la santé. La commission nationale d'éthique s'investit pour une société qui se donne les moyens d'aborder les sujets d'éthique de manière différenciée tout en étant consciente de la fragilité de la dignité humaine. C'est pourquoi elle ne perçoit pas l'éthique comme un frein à la recherche, mais comme partie intégrante et indispensable.

\section{La recherche sous la contrainte n'est pas éthique}

Il est prévu d'autoriser la recherche même si les personnes incapables de discernement s'y opposent, pour autant qu'elles profitent directement des travaux. Sur le plan éthique, il est injustifiable que, pour ce groupe, les intérêts scientifiques puissent primer sur la dignité humaine et la liberté personnelle.

D'ailleurs, une telle autorisation contreviendrait aux directives internationales. Une déroga- tion devrait reposer sur des arguments extrêmement solides - ce qui, aux yeux de la CNE, n'est pas le cas. La commission conçoit tout au plus certaines autorisations exceptionnelles.

\section{Les commissions sont plus que des organes de surveillance}

De ce projet se dégage un aspect positif: l'harmonisation des compétences attribuées aux commissions cantonales. La critique, quant à elle, porte sur la définition des commissions comme des organes de surveillance et d'examen uniquement.

Or, ces commissions doivent aussi tenir compte des principes éthiques et des directives internationales. Elles doivent examiner si une action donnée se justifie aux yeux de la société. Les aspects formels peuvent être également assumés par une autre instance. L'éthique s'attache à des considérations que la loi ne peut entièrement couvrir.

La prise de position est disponible sous: www.nek-cne.ch. 